\title{
Comportamiento isotrópico a altas presiones de arenas de relave con finos no plásticos
}

\author{
Isotropic behaviour of tailings sands with non-plastic fines at high pressures
}

Fecha de entrega: 20 de diciembre 2018

Fecha de aceptación: 27 de agosto 2019

\section{Camilo Córdova ${ }^{1,2}$, Felipe Ochoa ${ }^{2}$, Ramón Verdugo ${ }^{3}$, Roberto Olguín ${ }^{4}$, Miguel $\mathrm{Bravo}^{5}$ y Vicente Mercado ${ }^{6}$ \\ ${ }^{1}$ Golder Associates, Magdalena 181, piso 3, Las Condes, Región Metropolitana de Santiago, Chile, ccordova@golder.cl \\ ${ }^{2}$ Departamento de Ingenieria Civil, Universidad de Chile, Av. Blanco Encalada 2002, Santiago, Chile, fochoa@ing.uchile.cl \\ ${ }^{3}$ CMGI Ltda, Virginia Opazo 48, Santiago, Chile, rverdugo@cmgi.cl \\ ${ }^{4}$ GDE Consultores Geotécnicos, José Miguel Claro 070, Providencia, Región Metropolitana de Santiago, Chile, roberto.olguin@gmail.com \\ ${ }^{5}$ IDIEM, Universidad de Chile, Plaza Ercilla Poniente 883, Santiago, Chile, miguel.bravo@idiem.cl \\ ${ }^{6}$ Departamento de Ingeniería Civil y Ambiental, Universidad del Norte, km5 Vía Puerto Colombia, Barranquilla, Colombia, vmercado@uninorte.edu.co}

Se presenta un estudio experimental que examina el comportamiento triaxial isotrópico de arenas de relave en un amplio rango de presiones, desde $10 \mathrm{kPa}$ hasta 5 $M P a$, variando el contenido de finos de las muestras. Los resultados sugieren que la cantidad de finos aumentan la compresibilidad de las arenas de relave depositadas en estado suelto, generando cambios significativos en el índice de vacios al ser confinados en todo el rango de presiones estudiado. También se observa que el efecto de los finos en la compresibilidad disminuye con la disminución del indice de vacíos, exhibiendo incluso una rigidización de la muestra para los estados más densos de confección. Resultados de imagenología realizada post-ensayo sobre el material sugieren que la arena de relave presenta solo rotura de los cantos angulosos al ser consolidada a altas presiones; para índices de vacios bajos se observan diferencias en el contenido de finos de hasta un 4\% para la arena limpia. Esta diferencia disminuye al aumentar el contenido de finos de la arena, sugiriendo que la presencia de finos contribuye a la estabilidad de la estructura granular, redistribuyendo los esfuerzos interpartículas, disminuyendo el nivel de rotura.

Palabras clave: finos no plásticos, compresibilidad, altas presiones, relaves, consolidación
This article presents an experimental study that examines the isotropic triaxial behaviour of tailings sands in a wide range of pressures from $10 \mathrm{kPa}$ to 5 $M P a$, varying the fine content of the tested samples. The results suggest that the presence and quantity of fines have influence in the behaviour: there is an increase in the compressibility of tailings sands deposited in a loose state, generating significant changes in the void ratio when confined throughout the range of pressures studied. In addition, it is observed that the effect of the fines in the compressibility decreases with the decrease of the void ratio, even exhibiting stiffening of the sample for the densest conditions of confection. Results of imaging performed post-test on the material suggest that the tailings sand exhibits a slight breakage of its angular edges when consolidated at high pressures. For low void ratios, differences in fines content of up to $4 \%$ are observed for clean sand. This difference decreases when the fine content of the sand increases, suggesting that the presence of fines contributes to the stability of the granular structure, redistributing the interparticle stresses, decreasing the level of breakage.

Keywords: non-plastic fines, compressibility, high pressure, tailings, consolidation

\section{Introducción}

La caracterización y análisis del comportamiento de arenas se enfoca principalmente en el comportamiento de la estructura granular a niveles tensionales involucrados en proyectos de ingeniería convencionales, i.e., hasta $1 \mathrm{MPa}$. Dentro de este rango de tensiones, en particular el cambio del índice de vacíos según la presión de confinamiento está determinado por condiciones de formación del material, como el contenido de agua inicial (Hong et al., 2010), la 
temperatura (Baldi et al., 1988; Campanella y Mitchell, 1968), su cementación y envejecimiento (Schmertmann, 1983, 1984, 1991). Otro factor que influencia la compresibilidad de los materiales granulares a bajas presiones, por ejemplo, es el método de confección de las probetas (Casagrande, 1936; Santagata y Germaine, 2002; Schmertmann, 1955; Terzaghi y Peck, 1948).

Por otra parte, el desarrollo de infraestructura ha impulsado proyectos donde el nivel tensional sobre el suelo supera $1 \mathrm{MPa}$. Ejemplo de esto son los tranques de relave que, debido a la creciente industria minera, contemplan alturas de coronamiento sobre $\operatorname{los} 150 \mathrm{~m}$. A este nivel tensional el índice de vacíos varía a una tasa mucho menor con la presión de confinamiento, lo que se traduce en una disminución de la compresibilidad (Aplin et al., 1995; Athy, 1930). Los mecanismos que influencian este comportamiento a este nivel de presiones son la distribución de tensiones entre partículas, su reordenamiento granular, la rotura de partículas, y el efecto del tiempo, también conocido como creep (Barden, 1965; Mesri y Castro, 1987; Mesri y Godlewski, 1977). Por otro lado, la historia tensional y de formación de la muestra pierde relevancia en el comportamiento a estas presiones, independiente si la muestra es remoldeada o inalterada (Burland, 1990; Chilingar y Knight, 1960; Hong et al., 2012; Skempton, 1969; Terzaghi y Peck, 1948). En este contexto, en particular para arenas de relave, varios estudios sugieren que este tipo de arenas presentan mayor compresibilidad, particularmente para estados más sueltos y en la primera etapa de la consolidación (Maureira, 2012; Solans, 2010; Vargas, 2015).

Con respecto al aumento del contenido de finos, las arenas naturales evidencian un aumento en la compresibilidad del material al ser confinadas. La mayor compresibilidad se explica por estructuras "metaestables", en las cuales partículas de material fino están entre partículas de arena, tanto en el espacio de vacíos como a nivel de contacto. En particular, al ser cargado, el material fino en el contacto entre partículas de arena, al ser desplazado hacia el espacio intergranular, facilitarían el reacomodo de las partículas de arena (Yamamuro y Covert, 2001).

En el contexto de rotura de partículas, el contenido de finos influye en la distribución de las tensiones dentro del esqueleto granular. Estudios en arenas naturales muestran que un material bien graduado presenta menor rotura que uno mal graduado (Yamamuro y Covert, 2001). Lo anterior se debe a que, un material mal graduado, bajo una misma mineralogía, posee menores contactos entre partículas, lo que implica una mayor concentración de esfuerzos en estos contactos (Lee y Farhoomand, 1967). Otros estudios que analizan el fenómeno de rotura, en arenas de relave, en consolidación isótropa hasta una presión confinante de 5 $\mathrm{MPa}$, muestran que en estas arenas presentan un aumento en su contenido de finos de hasta un 3\% con respecto a su contenido de finos inicial (Maureira, 2012).

En este contexto, el presente trabajo muestra los resultados de 48 ensayos de consolidación triaxial, a presiones de confinamiento entre 1 y $5 \mathrm{MPa}$. Las probetas contienen 0 , 5,10 y $20 \%$ de finos, y densidades relativas de confección Dr de 15, 65 y 90\%. Además, se realizan ensayos de granulometría y microscopia óptica post consolidación para compararlas con el material intacto. Se analiza la evolución de la compresibilidad para un amplio rango de presiones de confinamiento. Además, se indaga en la posible ocurrencia de rotura particular.

\section{Equipos, materiales y programa experi- mental \\ Equipos}

Este estudio utilizó el equipo triaxial de altas presiones del Laboratorio de Sólidos y Medios Particulados de la Universidad de Chile, desarrollado por Solans (2010). El sistema tiene un marco de carga, sistemas de aplicación de carga axial, presión de cámara, contrapresión, cambio volumétrico, celda triaxial, así como instrumentación y sistemas de adquisición de datos.

\section{Materiales}

El plan experimental considera consolidaciones triaxiales isótropas, realizadas en muestras de arenas de relave, provenientes del tranque de relaves El Torito, perteneciente a la mina El Soldado. Esta arena es angular según lo observado en la Figura 1. El material original, con un contenido de finos $\mathrm{CF}=23 \%$, fue lavado con el fin de extraer las partículas finas, obteniéndose una arena de relave limpia, con un $1 \%$ de contenido de finos, como se observa en la Figura 2. Para desarrollar el plan experimental, la arena se mezcla con material fino, no 
plástico, para confeccionar materiales de 1, 5, 10 y $20 \%$ de contenido de finos NP.

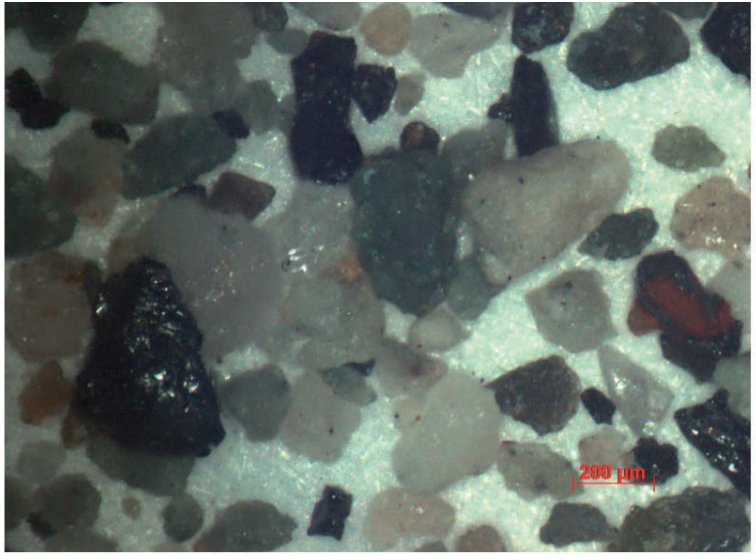

Figura 1: Arena de relaves $(\mathrm{CF}=1 \%)$, proveniente del tranque de relaves El Torito

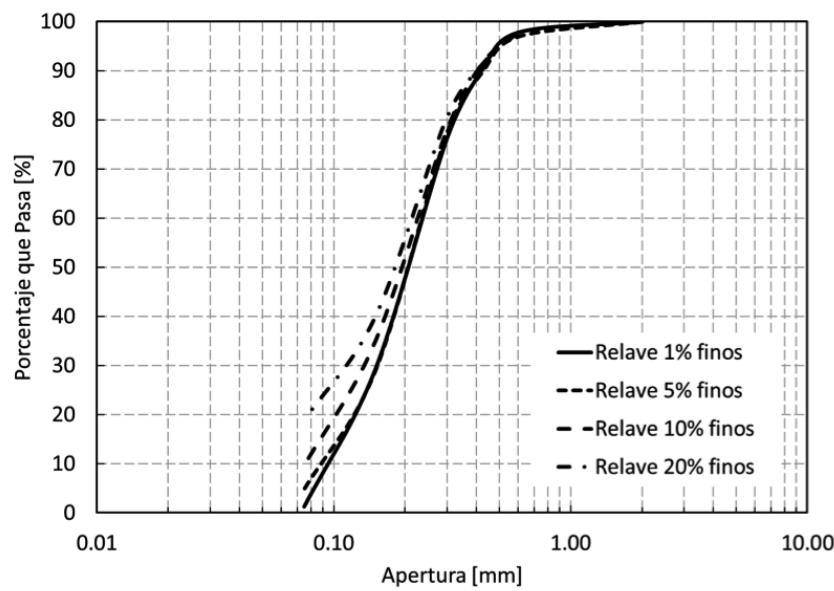

Figura 2: Curvas granulométricas de arenas ensayadas

\section{Programa experimental}

El programa experimental incluye 48 ensayos de consolidación isótropa, variando el contenido de finos de las muestras, la densidad relativa de confección y la presión de confinamiento. Para cada contenido de finos y densidad relativa, se llega a cuatro presiones de confinamiento indicadas en la Figura 3a) y 3b), se detiene el ensayo y se realizan granulometrías y microscopías por tamiz.

\section{Metodología}

La confección de probetas consideró el uso de la metodología de compactación húmeda (wet tamping), compactando en diez capas iguales en un molde de bronce de dimensiones conocidas. Finalizada la confección, la probeta se monta, se infiltra con $\mathrm{CO}_{2}$, agua destilada, se aplica una contrapresión de $200 \mathrm{kPa}$, y se verifica saturación a)

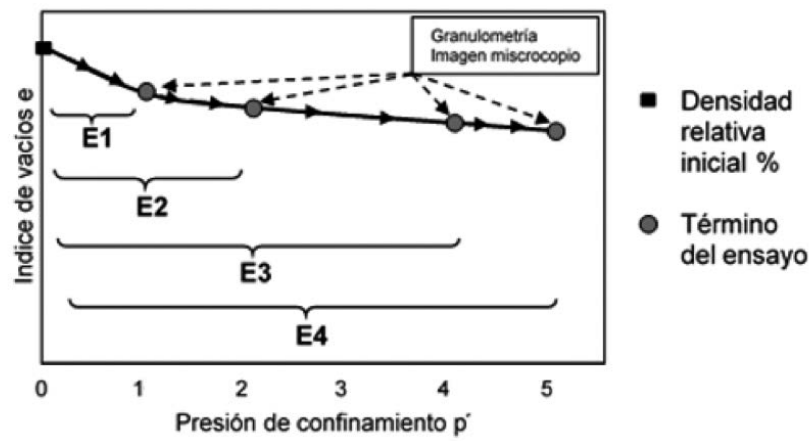

b)

Para un mismo contenido de finos inicial cf \%

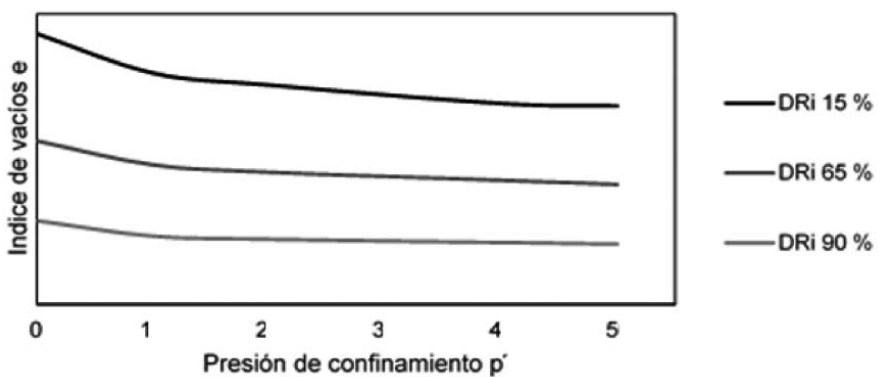

Figura 3: Esquematización del programa experimental. a) Desarrollo de la curva $e-p$ ' para cada material estudiado y b) esquema del desarrollo de densidades relativas para cada material (presión en $\mathrm{MPa}$ ).

midiendo el parámetro B de Skempton, para proceder a la consolidación, solo se ensayan probetas con un parámetro B igual o mayor a 0.95 .

El confinamiento aumenta en intervalos de $100 \mathrm{kPa}$, permitiendo el drenaje y estabilización del cambio volumétrico antes del siguiente incremento de carga. Se miden la presión de poros, deformación axial y el cambio volumétrico a los $30 \mathrm{~s}, 1 \mathrm{~min}, 2 \mathrm{~min}$ y $4 \mathrm{~min}$ de consolidación, hasta alcanzar la presión de confinamiento efectivo final deseada, monitoreando los cambios volumétricos del material ante la presión hidrostática.

Luego de la consolidación, se procede a la evaluación del índice de vacíos final del ensayo mediante el método utilizado por Verdugo e Ishihara (1996), el cual cuantifica el índice de vacíos de probetas saturadas a través del agua contenida en las muestras.

Posterior al ensayo de consolidación, el material se seca y se tamiza, para ser comparada con la granulometría del material sin ensayar. Además, se toman muestras de microscopía en el material retenido en cada uno de los tamices. En particular, esto se realiza en el material intacto, y para las consolidaciones realizadas hasta $2 \mathrm{MPa}$ y $5 \mathrm{MPa}$. 


\section{Resultados experimentales}

\section{Variación del índice de vacíos en consolidación}

El contenido de finos de un material tiene influencia en su comportamiento mecánico, así como en algunos de sus parámetros de estado que lo caracterizan. En la Figura 4 se muestran los resultados de los ensayos de vacíos máximos y mínimos, utilizando el método japonés (JGS, 2000) y de depositación lenta del material (ASTM D4254, 2000). El contenido de finos utilizado varía entre 1 y $50 \%$. Se adopta el método japonés en vez del método convencional (mesa vibratoria) para la estimación de densidad máxima, de manera de estandarizar los resultados con investigaciones realizadas anteriormente por otros autores en el mismo laboratorio con el mismo material (e.g. Maureira, 2012; Solans, 2010). Durante el ensayo, en particular para contenidos de finos altos sobre $30 \%$, no se tomó en consideración la migración de partículas finas dentro del cilindro metálico, ni se mantuvo registro de este fenómeno.

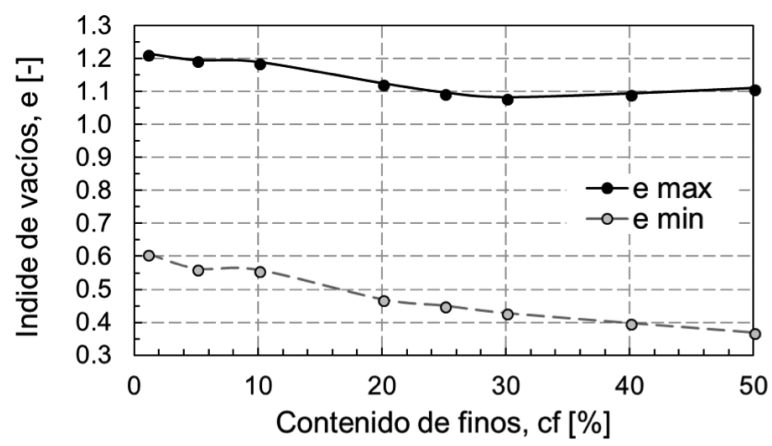

Figura 4: Resultados de los ensayos de densidad máxima (JGS, 2000) y mínima (ASTM D4254, 2000), para la arena de relave estudiada, variando el contenido de finos con respecto a la arena.

La Figura 5 muestra como varía el índice de vacíos $e$ al consolidar las muestras de manera isótropa para presiones de confinamiento desde $10 \mathrm{kPa}$ hasta $5 \mathrm{MPa}$. Se contrastan, para las mismas presiones, arenas de relave con contenidos de finos de $1,5,10$ y $20 \%$.

En la Figura 6, se muestra el índice de vacíos normalizado por el índice de vacíos inicial $e_{0}$ durante consolidación isótropa hasta una presión de confinamiento de $5 \mathrm{MPa}$. Se presentan, además, los resultados de ensayos de consolidación isótropa en arena natural de Ottawa, llevadas, también, hasta $5 \mathrm{MPa}$ de confinamiento y normalizadas por su índice de vacíos inicial $e_{0}$.

El índice de vacíos normalizado cuantifica cuanto más compresible es un material con respecto a otro, bajo los a)

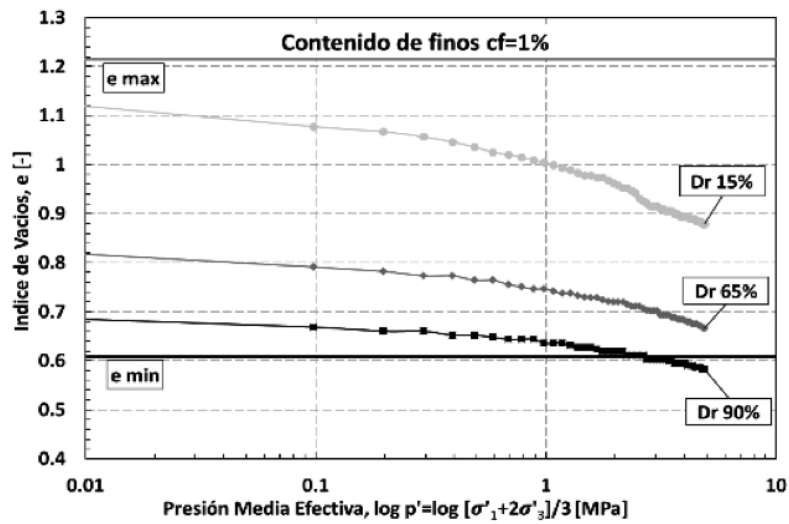

b)

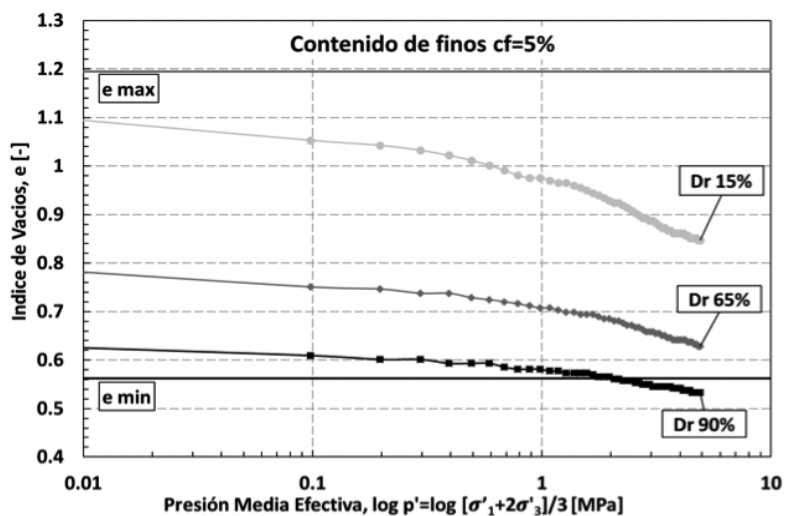

c)

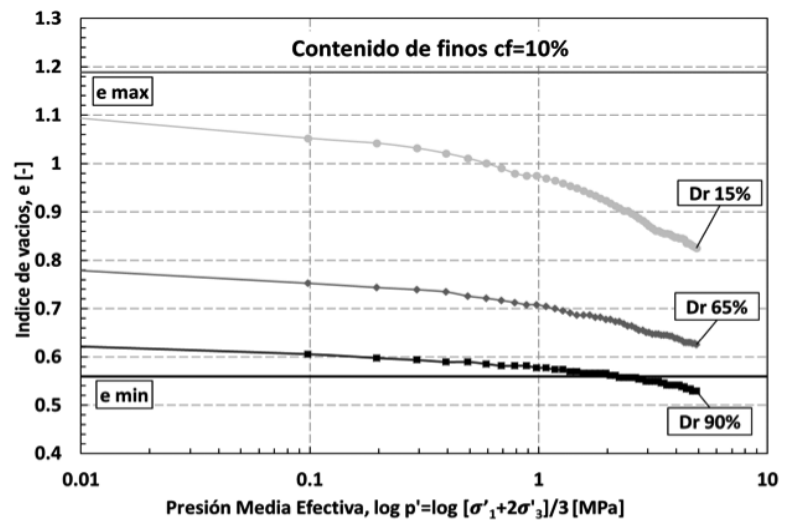

d)

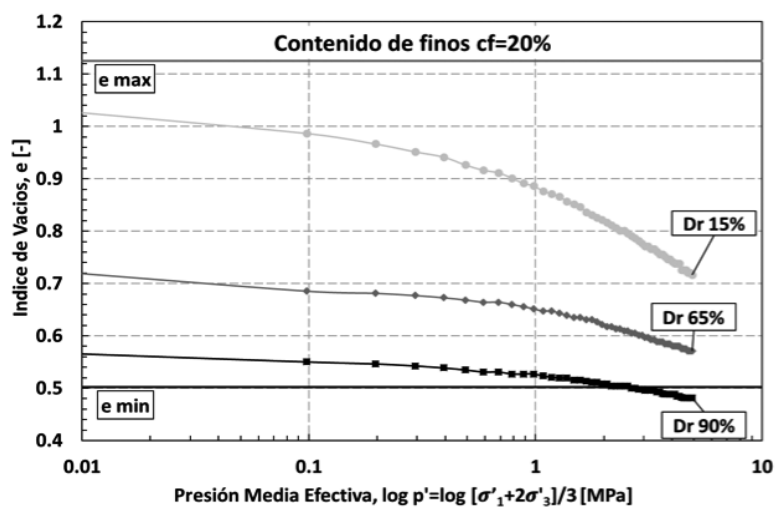

Figura 5: Variación del índice de vacíos $e$ en consolidación isótropa con la presión media $p^{\prime}$ y diferentes estados iniciales $\operatorname{Dr}=15,65$ y $90 \%$ para los distintos materiales estudiados con contenidos de finos CF de: a) $1 \%$, b) $5 \%$, c) $10 \%$ y d) $20 \%$ 
mismos estados de carga. Las Figura 6a y $6 \mathrm{c}$ muestran que, las arenas de relave presentan un mayor cambio en su índice de vacíos al estar afectados por cargas isotrópicas, en relación con las arenas de Ottawa redondeadas. Además, para los estados más sueltos de las arenas de relave estudiadas ( $\mathrm{Dr}=15$ y 65\%), se observa que, al aumentar el contenido de finos de las muestras, estas evidencian un mayor cambio en su índice de vacíos durante consolidación isótropa, para todo el rango de presiones estudiado.

a)

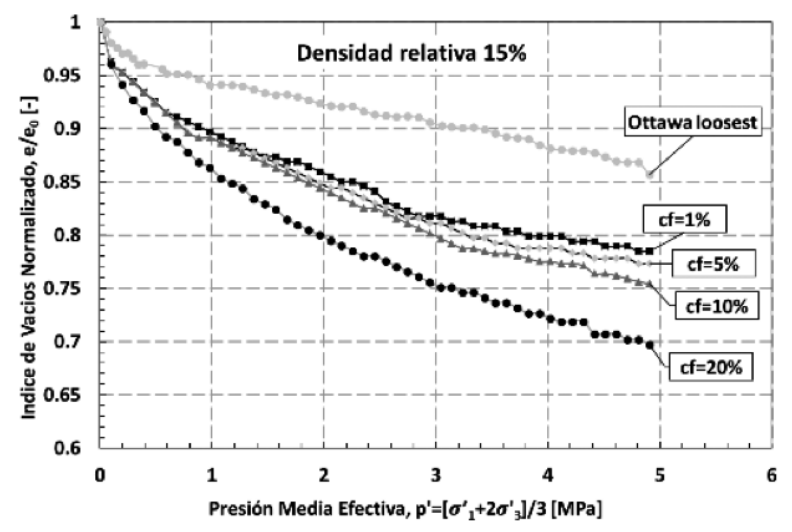

b)

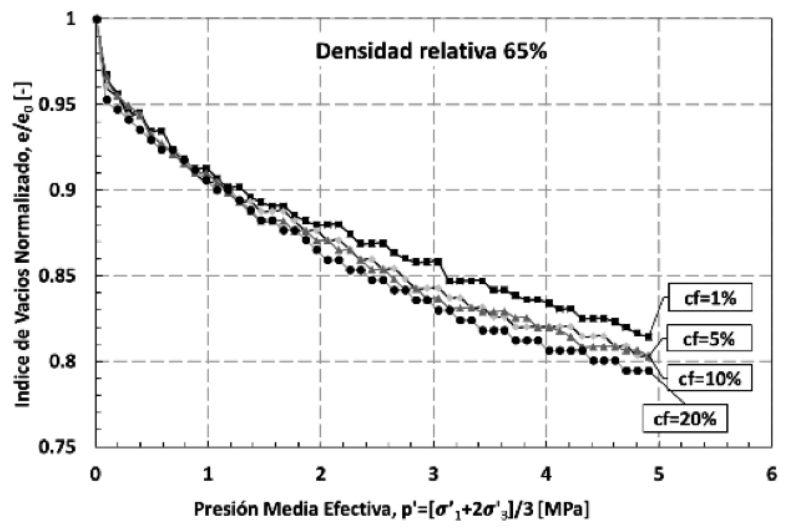

c)

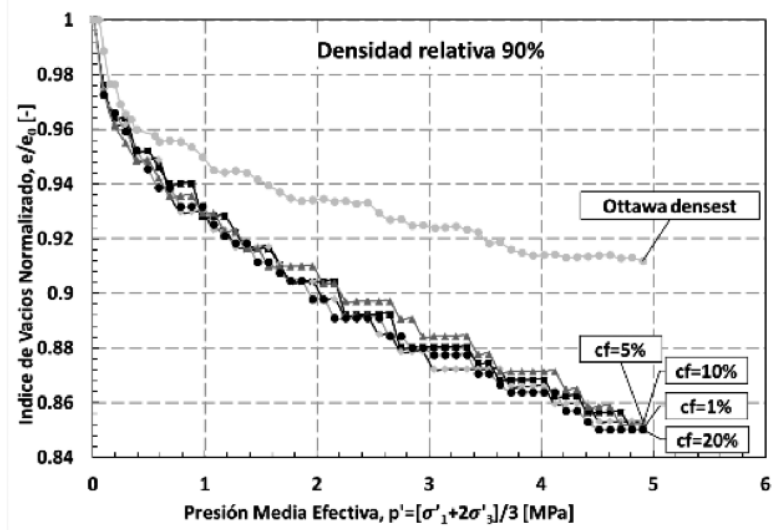

Figura 6: Variación del índice de vacíos $e$ en consolidación isótropa normalizado por el índice de vacíos inicial $e_{0}$ en relación a la presión media $p$ ' y para distintos materiales estudiados $(\mathrm{CF}=1,5,10$ y $20 \%)$ para tres estados iniciales $\mathrm{Dr}$ de: a) $15 \%$, b) $65 \%$ y c) $90 \%$

\section{Rotura de partículas}

Se utiliza el factor de rotura de partículas $B_{10}$, propuesto por Lade y Yamamuro (1996) y definido en (1). Este parámetro entrega valores en el rango de 0 a 1 y representa el porcentaje de variación que tiene el 10\% más fino de la muestra de material ensayado.

$$
B_{10}=1-\frac{D_{10 f}}{D_{10 i}}
$$

Donde $D_{10 f}$ es el tamaño de partícula para el cual el $10 \%$ de la muestra ensayada es más fina y $D_{10 i}$ es el tamaño de partícula donde el $10 \%$ de la muestra original es más fina.

En la Figura 7 se contrastan todas las densidades relativas analizadas en este estudio, variando el contenido de finos para los ensayos de consolidación isótropa.

\section{Discusión}

$e_{\text {max }} \mathrm{y} e_{\text {min }}$

La curva conformada por los estados más sueltos llega a un valor mínimo $e_{\max }=1.082$, luego de esto, tiende a aumentar, según lo indicado en la Figura 4. Esta observación concuerda con el comportamiento de arenas naturales con finos no plásticos exhibido por estudios previos (Lade et al., 1998), quienes observaron que el índice de vacíos máximo disminuye inicialmente hasta un porcentaje que puede oscilar entre $\mathrm{CF}=20$ y $40 \%$, para luego volver a aumentar. Lo anterior ocurre mientras las partículas finas puedan alojarse entre los espacios intersticiales. Sin embargo, cuando el contenido de finos aumenta, estos se empiezan a alojar entre las partículas de arena, al nivel de su contacto, separándolas, como se esquematiza en la Figura 8. Esto concuerda con lo presentado por Yamamuro y Covert (2001), quienes observaron que las partículas finas se acomodan como una estructura denominada "metaestable", al alojarse entre los contactos de las partículas de arena separándolas.

Al analizar la curva conformada por los estados más densos en la Figura 4, se observa que esta es decreciente para todos los porcentajes de finos analizados. Esto se debe a que, por la naturaleza angulosa de las partículas de arena de relave y la alta energía de compactación, los finos, de naturaleza angular, también tienden a acomodarse en los espacios vacíos entre las arenas, disminuyendo el índice, como se esquematiza en la Figura 9. 
a)

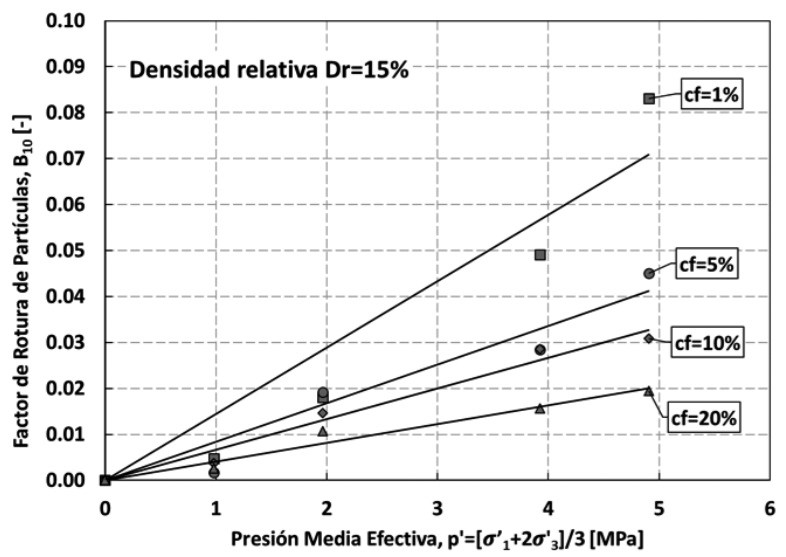

b)

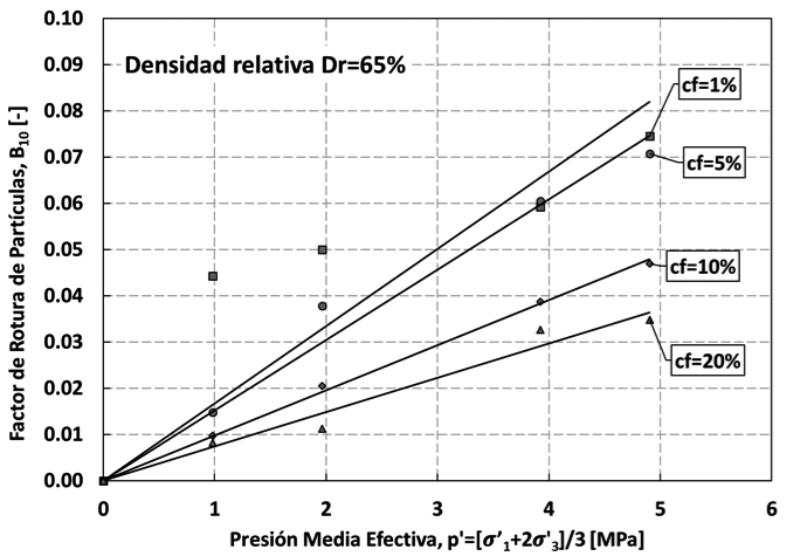

c)

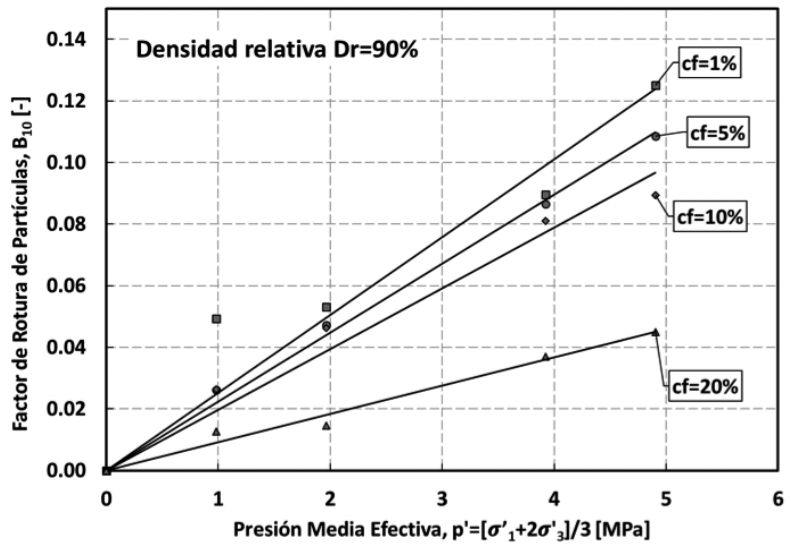

Figura 7: Variación del factor de rotura de partículas $B_{10}$ (Lade y Yamamuro, 1996) con la presión media efectiva $p$ ' para muestras de densidad relativa de confección de: a) $15 \%$, b) $65 \%$ y c) $90 \%$

\section{Compresibilidad}

Los resultados obtenidos de las consolidaciones hasta 5 $\mathrm{MPa}$, para los estados más sueltos y más densos $(\mathrm{Dr}=15 \%$ y $90 \%$ ), muestran una amplia región de estados posibles de índices de vacíos para todos los materiales estudiados. Esto se debe a la naturaleza irregular/angulosa de estas arenas, que permite formar estructuras con índices de vacíos mayores en comparación con arenas naturales que pueden

\section{Estado más suelto}
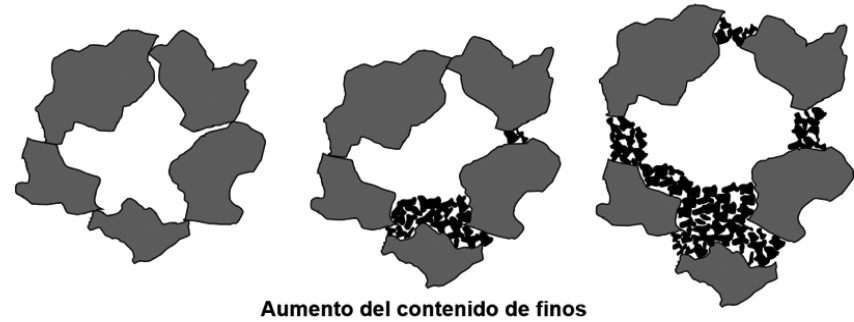

Aumento del contenido de finos

Figura 8: Esquema del mecanismo de ordenamiento del estado más suelto para la arena de relave al aumentar su contenido de finos.

\section{Estado más denso}
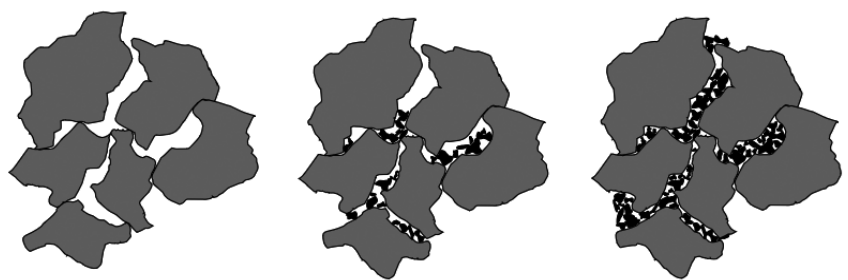

Aumento del contenido de finos

Figura 9: Esquema del mecanismo de ordenamiento del estado más denso para la arena de relave al aumentar su contenido de finos.

presentar más redondeo en su superficie. Lo anterior se traduce en una mayor capacidad de reordenamiento de partículas al ser consolidada.

Se observa en los resultados de consolidaciones isótropas que, para los ensayos realizados a densidades relativas de confección de $90 \%$, llevadas por sobre los 2 MPa de presión de confinamiento, se alcanzan índices de vacíos menores al valor mínimo obtenido en los ensayos de densidad máxima entregados por el método japonés (JGS, 2000), para todos los materiales ensayados. A niveles de tensión elevados, es posible alcanzar estados de ordenamientos de partículas más compactas, no alcanzables con los métodos estandarizados de compactación que determinan el índice de vacíos mínimo.

Con respecto a los resultados presentados en las Figuras 5a-5d, donde se muestra el cambio de índice de vacíos durante consolidación isótropa, se observa un aumento de compresibilidad, al aumentar el contenido de finos. Al normalizar el índice de vacíos por el índice de vacíos inicial, Figuras 6a-6c, se evidencia de mejor forma el cambio de compresibilidad de las muestras ensayadas. Al 
comparar la arena con $20 \%$ de contenido de finos con la arena lavada $(\mathrm{CF}=1 \%)$, existe un $10 \%$ de diferencia entre el índice de vacíos final con el índice de vacíos inicial, en ensayos de consolidación hasta $5 \mathrm{MPa}$.

Esta diferencia se va haciendo cada vez menos notoria al aumentar la densidad relativa de confección de las muestras. Este comportamiento es debido a que, para los estados más densos, los finos se acomodarían entre la estructura granular, con una metaestructura ya desplazada, acomodada y estable (Yamamuro y Covert, 2001), que no aportaría en el reordenamiento de las partículas de arena, incluso restringiendo su cambio. Para el caso de los estados más sueltos, los finos tendrían gran influencia en la compresibilidad del material. La estructura metaestable aportaría en el reordenamiento de las partículas de arena al ser consolidadas de manera isotrópica, como se esquematiza en la Figura 10.

Para cuantificar la compresibilidad de las arenas estudiadas a altas presiones, por sobre $1 \mathrm{MPa}$, con diferentes dosificaciones de contenido de finos, se propone el coeficiente de compresión, $C_{\mathrm{c} 10}$, el subíndice indica que es la compresibilidad por sobre $1 \mathrm{MPa}$, que es diferente al utilizado normalmente en compresión oedométrica a bajas presiones. Los subíndices utilizados para el índice de vacío $e_{x}$ y para la presión de confinamiento $p_{x}^{\prime}$ indican la presión de confinamiento en que fueron evaluados.

$$
C_{c 10}=\frac{e_{50}-e_{10}}{\log \left(p_{50}^{\prime}\right)-\log \left(p_{10}^{\prime}\right)}
$$

La Figura 11 muestra los valores del coeficiente de compresión para la arena de relave con diferentes dosificaciones de contenido de finos ensayadas hasta 5 MPa, para las densidades de confección de 15\%,65\% y $90 \%$. Este parámetro muestra el aumento gradual de la compresibilidad para los estados más sueltos. Finalmente, para el caso de las densidades relativas de $90 \%$, los resultados muestran un aumento en la rigidez volumétrica, presentándose cada vez menores variaciones en el índice de vacíos al aumentar el contenido de finos de las muestras. Los finos ya acomodados entre los espacios intergranulares tenderían a restringir el reacomodo de las partículas más grandes, lo que explica el leve aumento en la rigidez volumétrica.
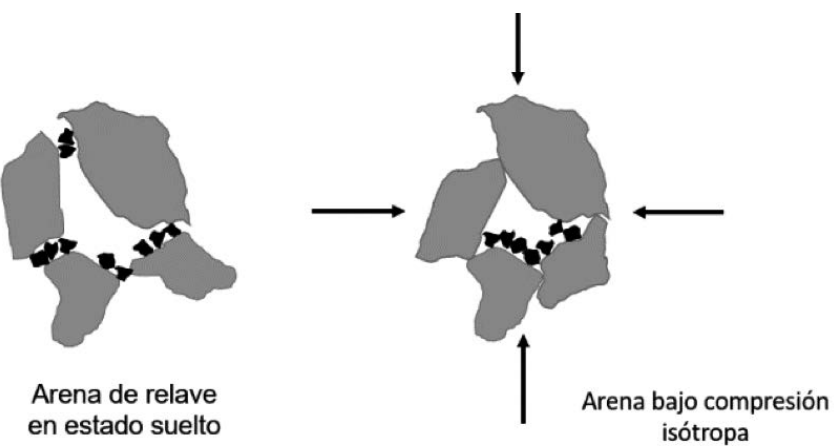

Arena de relave en estado suelto isótropa

Figura 10: Mecanismo de metaestructura movilizada para estados sueltos de arena de relave al ser consolidada

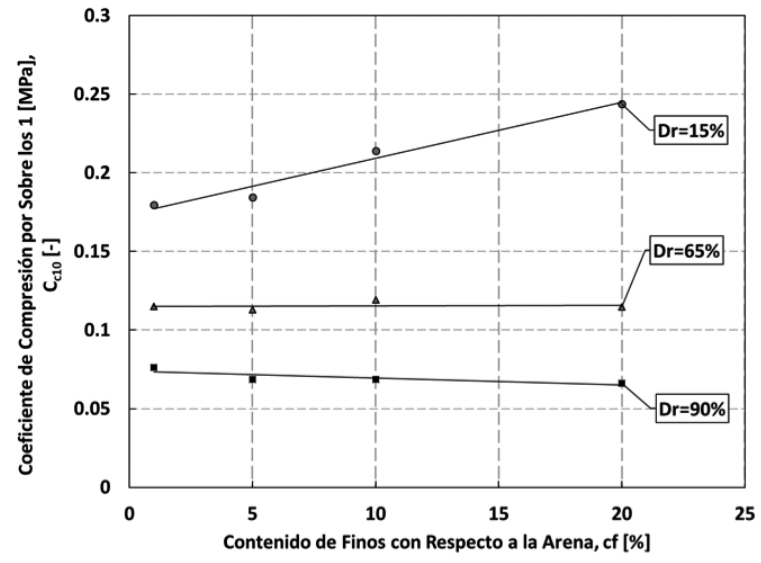

Figura 11: Variación del coeficiente de compresión $C_{c 10}$, para p' mayor a $1 \mathrm{MPa}$ al aumentar la cantidad de finos, para las densidades relativas estudiadas $(\mathrm{Dr}=15 \%, 65 \%$ y $90 \%)$.

\section{Evidencia de pulido de cantos}

Para analizar la influencia que tiene la densidad relativa en la rotura, se comparó la diferencia entre las granulometrías pre y post consolidación, usando el factor de rotura de partículas $B_{10}$, indicada en (1), este factor muestra la variación de tamaño en el $10 \%$ más fino de las muestras. Se utiliza este factor debido a que estas arenas no muestran una rotura del grano completo, sino más bien un pulido de sus cantos angulosos (Maureira, 2012), generando material fino. Las muestras ensayadas de mayor densidad relativa presentan mayores valores del factor de rotura, lo cual concuerda con los resultados de investigaciones anteriores (Solans, 2010), en el mismo tipo de arena, aun cuando el contenido de finos es diferente. Esto se explica por la naturaleza angulosa de las arenas de relave que permite un estado suelto con grandes índices de vacíos, lo que resulta en una gran capacidad de reordenamiento sin que se rompan los cantos angulosos. Este comportamiento 
es contrario a lo visto en la literatura técnica en arenas naturales redondeadas, como se indica en trabajos realizados por Bopp y Lade (1997) y Hardin (1985), donde se observa que, al existir menos contactos entre partículas, las arenas en estados sueltos muestran mayor rotura en arenas naturales.

El comportamiento diferente para arenas de relaves con respecto a arenas naturales se puede explicar desarrollando una hipótesis en torno a la forma de las partículas. Si bien, al tener densidades mayores existe un mejor acomodo de partículas y mayor número de puntos de contacto que distribuye más uniformemente los esfuerzos efectivos, estos son de un área mucho menor, frágil e irregular que generarían concentración de tensiones generando un quebramiento/pulido de los cantos angulosos.

Las Figuras $7 \mathrm{~b}$ y $7 \mathrm{c}$ muestran que para las densidades relativas $\mathrm{Dr}=65 \%$ y $90 \%$, se observa un aumento considerable de la rotura para las probetas con $1 \%$ de contenido de finos a $1 \mathrm{MPa}$ de presión de confinamiento. De manera diferente sucede para la muestra confeccionada a una densidad relativa de $15 \%$, en el que el valor del factor de rotura aumenta paulatinamente, casi no evidenciando rotura inicialmente. Esto sugiere que, para estados más sueltos existirá más espacio para el reacomodo de partículas retardando la rotura de los cantos angulosos producto de un trabamiento granular.

Con el fin de presentar la influencia que tiene el contenido de finos en la rotura de partículas se propone utilizar el área bajo la curva en el plano $B_{10}-p^{\prime}$, definida por la expresión (3).

$$
\Delta A_{B_{10}}=\frac{1}{2}\left(\frac{p_{i+1}^{\prime}}{p_{\max }}-\frac{p_{i}^{\prime}}{p_{\max }}\right)\left(B_{10 \mathrm{i}+1}+B_{10 \mathrm{i}}\right)
$$

Este factor resume la información obtenida de rotura de las muestras, para cada nivel tensional analizado, desde $10 \mathrm{kPa}$ a $5 \mathrm{MPa}$, especificados en los subíndices de la ecuación. Los resultados mostrados en la Figura 12 exhiben una disminución de la rotura de partículas con el aumento del contenido de finos. Esta tendencia sugiere que se produce un aumento de los contactos entre partículas al aumentar el contenido de finos, distribuyendo de mejor manera las tensiones efectivas y disminuyendo el nivel de rotura.

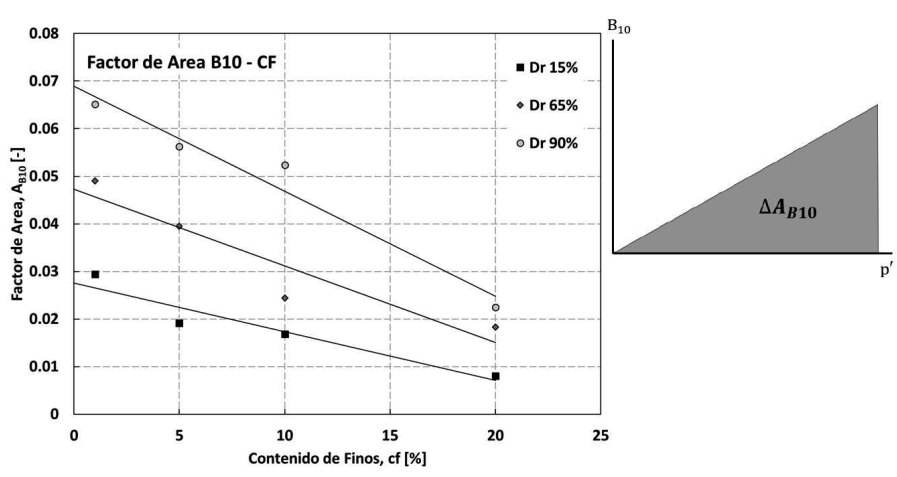

Figura 12: Factor de área $\mathrm{B}_{10}$ al variar el contenido de finos para las densidades vistas en este estudio

La Figura 7c muestra que los ensayos realizados en probetas de densidad relativa de confección de $90 \%$ exhibieron mayor variación del contenido de finos en relación con las demás densidades estudiadas. Se destaca que, para la arena lavada, con un $1 \%$ de contenido de finos inicial, la variación de este porcentaje fue de un $4 \%$ para la muestra más densa. Esta diferencia llega a ser de 3\% para los ensayos con $10 \%$ de contenido de finos inicial y de un $1 \%$ para los ensayos de $20 \%$ de contenido de finos inicial.

Complementando el análisis granulométrico, post consolidación isótropa, se tomaron imágenes de microscopio por tamiz, para las muestras llevadas hasta una presión de confinamiento de $2 \mathrm{MPa}$ y $5 \mathrm{MPa}$, estas contrastadas con la arena sin ensayar. Esto se realiza para identificar si existe rotura, comparando la arena sin ensayar con la arena post ensayo. Se observa una sutil tendencia de estas a ser más redondeadas si el contenido de finos de la muestra es menor, como se observa en la Figura 13. Esto sugiere que la rotura vista en los ensayos de consolidación corresponde a la rotura de los cantos angulosos propia de la arena de relaves.
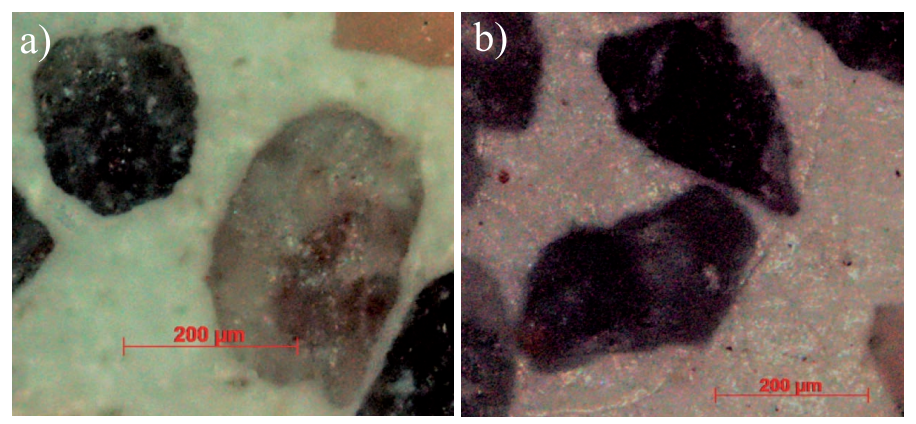

Figura 13: Comparación de la forma de partículas de arena a una misma densidad relativa $\mathrm{Dr}=90 \%$, variando el contenido de finos: a) arena ensayada hasta $5 \mathrm{MPa}$ con $\mathrm{CF}=1 \%$ y b) arena ensayada hasta $5 \mathrm{MPa}$ con contenido de finos de $20 \%$. 


\section{Conclusiones}

Los resultados de este programa experimental sugieren que el aumento del porcentaje de finos no plásticos aumenta la compresibilidad de las arenas de relave para densidades relativas bajas con $\mathrm{Dr}=15 \%$. Las arenas con un $20 \%$ de contenido de finos mostraron una variación volumétrica de hasta un $10 \%$ mayor que la arena lavada $(\mathrm{CF}=1 \%)$. El resultado de esto se ve en una gran variación de la densidad relativa de las muestras luego del confinamiento. La presencia de finos entre los contactos de las partículas de arena de relave en estado suelto, junto con la naturaleza angular de estas, hace que existan grandes espacios entre partículas, que facilitarían el reordenamiento al ser consolidadas. Lo anterior sugiere que la compresibilidad de la arena de relave, a altas presiones, se ve significativamente influenciada por la densidad relativa inicial y la cantidad de finos en ellas. Por otro lado, el contenido de finos no plásticos para muestras densas $(\mathrm{Dr}=90 \%)$, tiende a disminuir levemente la variación volumétrica en consolidación isótropa, haciéndolas menos compresibles.

El porcentaje de finos en estas arenas muestra tener influencia en el nivel de rotura de partículas en ensayos de consolidación. Los resultados sugieren que la rotura presentada en arenas de relave es cada vez mayor al aumentar la densidad relativa de confección y disminuye al aumentar el contenido de finos. Esto se repite para todas las densidades relativas estudiadas, observándose una diferencia en el parámetro de rotura $B_{10}$ de un $6 \%$, para la arena de relave con $20 \%$ de contenido de finos, en relación con la arena lavada $(\mathrm{CF}=1 \%)$, en muestras en estado suelto $(\mathrm{Dr}=15 \%)$. Este patrón también se evidencia en muestras densas ( $\mathrm{Dr}=90 \%$ ), exhibiendo una diferencia de hasta un $8 \%$ en el parámetro, para las muestras con $20 \%$ de contenido de finos, en relación con la arena lavada $(\mathrm{CF}=1 \%)$.

Para los estados más sueltos $(\mathrm{Dr}=15 \%)$, se puede ver que el desarrollo del nivel de rotura $B_{10}$, dado por el parámetro de rotura, es casi nulo hasta presiones de confinamiento de $1 \mathrm{MPa}$, alcanzando un valor máximo de $0.5 \%$ en la arena lavada $(\mathrm{CF}=1 \%)$. Por otro lado, para las muestras más densas $(\mathrm{Dr}=90 \%)$, para la misma arena lavada $(\mathrm{CF}=1 \%)$, evidencian diferencias de hasta un $5 \%$ en la fracción más fina en las muestras, obtenidas con el parámetro de rotura $B_{10}$, para un mismo nivel de confinamiento de $1 \mathrm{MPa}$.
Se propone un mecanismo para explicar la baja tasa de rotura de partículas en las muestras más sueltas, a bajas presiones de confinamiento. La naturaleza angulosa de las partículas de arena tiene la capacidad de formar estructuras estables con grandes espacios entre partículas. Estos espacios le darían a este tipo de arenas una gran capacidad de reordenamiento antes de que se produzca trabazón mecánica que genere rotura. Este efecto, combinado con la mejora en la distribución de esfuerzos efectivos entre partículas y al efecto positivo en el reordenamiento de partículas de arenas al agregar finos, retarda y disminuye el pulido de los cantos angulosos.

\section{Referencias}

Aplin, A.C., Yang, Y. and Hansen, S. (1995). Assessment of $B$ the compression coefficient of mudstones and its relationship with detailed lithology. Marine and Petroleum Geology 12(8), 955-963

ASTM D4254 (2000). Standard test methods for minimum index density and unit weight of soils and calculation of relative density. ASTM International, West Conshohocken, PA, USA

Athy, L.F. (1930). Density, porosity, and compaction of sedimentary rocks. AAPG Bulletin 14(1), 1-24.

Baldi, G., Hueckel, T. and Pellegrini, R. (1988). Thermal volume changes of the mineral-water system in low-porosity clay soils. Canadian Geotechnical Journal 25(4), 807-825

Barden, L. (1965). Consolidation of clay with non-linear viscosity. Géotechnique 15(4), 345-362

Bopp, P.A. and Lade, P.V. (1997). Effects of initial density on soil instability at high pressures. Journal of Geotechnical and Geoenvironmental Engineering 123(7), 671-677

Burland, J.B. (1990). On the compressibility and shear strength of natural clays. Géotechnique 40(3), 329-378

Campanella, R.G. and Mitchell, J.K. (1968). Influence of temperature variations on soil behavior. Journal of the Soil Mechanics and Foundations Division 94(3), 609-734

Casagrande, A. (1936). The determination of the preconsolidation load and its practical significance. $1^{\text {st }}$ International Conference on Soil Mechanics and Foundation Engineering, Harvard University, Cambridge, USA, vol. 3, 60-64 
Chilingar, G.V. and Knight, L. (1960). Relationship between pressure and moisture content of kaolinite, illite, and montmorillonite clays. AAPG Bulletin 44(1), 101-106

Hardin, B.O. (1985). Crushing of soil particles. Journal of Geotechnical Engineering 111(10), 1177-1192

Hong, Z.S., Zeng, L.L., Cui, Y.J., Cai, Y.Q. and Lin, C. (2012). Compression behaviour of natural and reconstituted clays. Géotechnique 62(4), 291-301

Hong, Z.S., Yin, J. and Cui, Y.J. (2010). Compression behaviour of reconstituted soils at high initial water contents. Géotechnique 60(9), 691-700

JGS (2000). Test methods for minimum and maximum densities of sands. Soil testing standards. Japanese Geotechnical Society JGS, 136-138 (in Japanese)

Lade, P. and Yamamuro, J. (1996). Undrained sand behavior in axisymmetric tests at high pressures. Journal of Geotechnical Engineering 122(2), 120-129

Lade, P.V., Liggio, C.D. and Yamamuro, J.A. (1998). Effects of non-plastic fines on minimum and maximum void ratios of sand. Geotechnical Testing Journal 21(4), 336-347

Lee, K.L. and Farhoomand, I. (1967). Compressibility and crushing of granular soil in anisotropic triaxial compression. Canadian Geotechnical Journal 4(1), 68-86

Maureira, S. (2012). Respuesta cíclica de arena de relaves en un amplio rango de presiones. Tesis de magíster, Universidad de Chile

Mesri, G. and Castro, A. (1987). $C_{\alpha} / C_{\mathrm{c}}$ concept and $K_{0}$ during secondary compression. Journal of Geotechnical Engineering 113(3), 230-247

Mesri, G. and Godlewski, P.M. (1977). Time and stress compressibility inter relationship. Journal of the Geotechnical Engineering Division 103(5), 417- 430
Santagata, M. and Germaine, J. (2002). Sampling disturbance effects in normally consolidated clays. Journal of Geotechnical and Geoenvironmental Engineering 128(12), 997-1006

Schmertmann, J.H.(1983). Asimple question about consolidation. Journal of Geotechnical Engineering 109(1), 119-122

Schmertmann, J.H. (1984). Closure to 'A simple question about consolidation'. Journal of Geotechnical Engineering 110(5), 673

Schmertmann, J.H. (1991). The mechanical aging of soils. Journal of Geotechnical Engineering 117(9), 1288-1330

Schmertmann, J.H. (1955). The undisturbed consolidation behavior of clay. Transactions of the American Society of Civil Engineers 120(1), 1201-1233

Skempton, A.W. (1969). The consolidation of clays by gravitational compaction. Quarterly Journal of the Geological Society 125(1-4), 373-411

Solans, D. (2010). Equipo triaxial monótono y cíclico de altas presiones y su aplicación en arenas de relave. Tesis de magíster, Universidad de Chile

Terzaghi, K. and Peck, R. B. (1948). Soil mechanics in engineering practice. Wiley, New York, USA

Vargas, M. (2015). Efecto acoplado de la presión de confinamiento y el corte estático inicial en la resistencia cíclica de arena de relaves. Tesis de magíster, Universidad de Chile

Verdugo, R. and Ishihara, K. (1996). The steady state of sandy soils. Soils and Fundations 36(2), 81-91

Yamamuro, J.A. and Covert, K.M. (2001). Monotonic and cyclic liquefaction of very loose sands with high silt content. Journal of Geotechnical and Geoenvironmental Engineering 127(4), 314-324 